\title{
Outage Analysis of Energy Harvested Direct/Relay-Aided Device-to-Device Communications in Nakagami Channel
}

\author{
Shahriar Shirvani Moghaddam, Senior Member, IEEE
}

\begin{abstract}
In this paper, a low-complexity closed-form formula for the outage probability of the energy-harvested direct and decode-and-forward (DF) relay-aided underlay device-to-device (D2D) communications in Nakagami fading channel is derived. By proposing a new procedure, the power splitting factor in simultaneous wireless information and power transfer (SWIPT) energy-harvesting system is easily found such that the transmit power of the transmitter node in the direct and the relay node in a relay-aided D2D communications is fixed in a pre-defined value. Using the proposed procedure, the obtained closed-form expression is valid for both energy-harvested and non-energyharvested scenarios. This formula is based on n-point generalized Gauss-Laguerre and m-point Gauss-Legendre solutions. It is shown that $n$ is more effective than $m$ for reducing the formula complexity. In addition to a good agreement between the simulation results and numerical analysis based on normalized mean square error $(\mathrm{NMSE})$, it is indicated that $(\mathrm{n}, \mathrm{m})=(1,4)$ and $(\mathrm{n}, \mathrm{m})=(1,2)$ are the appropriate choices, respectively for $0.5 \leq \mu$ $<0.7$ and $\mu \geq 0.7$, where $\mu$ is the fading shape factor. As shown in this investigation, increasing the average distance between the D2D pair and cellular user introduces lower interference, which is the reason for decreasing the outage probability. Furthermore, it is clear that increasing the Nakagami fading shape factor is the reason for decreasing the depth of fading, its fluctuations and the outage probability.
\end{abstract}

Index Terms-Device to device (D2D) communications, Decodeand-forward (DF), Energy harvesting (EH), Simultaneous wireless information and power transfer (SWIPT), Gauss-Legendre/GaussLaguerre, Nakagami, Outage probability.

\section{INTRODUCTION}

$\mathrm{T}$ he limitation of radio resources and the rapid growth of internet and telecommunication applications, make the optimal usage of radio resources essential. One of the main strategies for reusing radio resources is the device to device (D2D) communications between two nearby users. The D2D pairs directly or through relay, overlay or underlay, in-band or out-band, transmit and receive the signal [1].

Manuscript received March 13, 2018; revised July 15, 2018. Date of publication October 15, 2018. The associate editor Prof. Francesca Vatta has been coordinating the review of this manuscript and approved it for publication.

S. Shirvani Moghaddam is with the Faculty of Electrical Engineering, Shahid Rajaee Teacher Training University, Tehran, 1678815811, Iran (e-mail: sh_shirvani@sru.ac.ir).

Digital Object Identifier (DOI): 10.24138/jcomss.v14i4.490
In the field of the D2D communications underlaying cellular network, first the communications mode should be selected between conventional cellular, relay-assisted cellular, direct D2D, and relay-assisted (or relay-aided) D2D modes. In the cellular mode, using a two-hop link, the cellular user sends its signal to the base transceiver station (BTS) in the uplink and BTS forwards the enhanced received signal to the target cellular user in the downlink. When the transmitter and receiver nodes of a link are close to each other, a direct D2D communications can be set to improve the throughput and decrease the delay. When the distance between transmitter and receiver (TX and $\mathrm{RX}$ ) nodes of a direct D2D link is greater than a predefined value depending on the signal to interference plus noise ratio (SINR) threshold level, the signal can experience a two-hop link, first from the D2D transmitter to the relay and then from the relay to the $\mathrm{D} 2 \mathrm{D}$ receiver. The relay has a limited coverage area much lower than a BTS but it can handle the signals between two distant D2D users, which improves the total achievable throughput especially for the users near to the cell boundary. Several relaying strategies have been proposed, including amplify-and-forward (AF) and decode-and-forward (DF) [2-6]. In DF, which is mentioned in this research, the relay decodes the received signal, re-encodes it, and finally sends it to the RX node of the D2D pair [2].

One of the main procedures for optimizing underlay in-band D2D communications is minimizing the outage probability (the probability that the received SINR is less than the threshold level) considering the impacts of additive white Gaussian noise (AWGN), interference and multipath fading components in a joint manner. In order to optimize the system performance in a low-complexity procedure, it is needed to have low-complexity closed-form expressions for the outage probability in the optimization problems.

Research works [2, 3], obtained closed-form formulas for the outage probability of direct underlay/overlay D2D communications in a Rayleigh channel. In Ref. [7], the exact formulas for the outage probability of in-band direct D2D communications underlying cellular network in a Rayleigh channel were proposed. The proposed model considers two radio resources reuse scenarios, one-reuse and two-reuse. The authors of [8] exploited stochastic geometry, Laplace transforms, and probability density function (pdf) to calculate the outage probability in a tractable manner. 
Ref. [9] considered $\kappa-\mu$ and $\eta-\mu$ distributions for the fading behaviour in D2D communications. By using stochastic geometry, the spectral efficiency and the outage probability of D2D networks under generalized fading conditions were investigated. Recently, Ref. [10] derived an expression for the outage probability of underlay direct D2D communications in a Suzuki channel, which jointly considers multipath Rayleigh fading and shadowing components.

Recently, Ref. [11] derived two closed-form expressions for the outage probability of non-orthogonal in-band AF and DF relay-aided D2D communications in Rayleigh channels. According to the numerical analyses of this investigation, it was indicated that in the low-noise regime, AF outperforms DF while they perform very close to each other in the moderate noise level and DF outperforms AF in the high-noise power. In [12], an exact outage probability expression for DF cognitive dual-hop system in Nakagami-m fading channel was derived. The authors in [13] derived the exact and asymptotic closedform expressions for the outage probability of underlay cognitive DF relay network with the Nth best relay selection scheme over Nakagami-m fading channels. In [14], the authors derived exact closed-form expressions and tight approximations for the outage probability of DF relay-assisted D2D link under asymmetrical and symmetrical cases. In [15], the coverage probability of both the cellular users (CUs) and the direct or relay-assisted D2D users was analyzed.

The theoretical and numerical analyses and formulations for the outage probability of the direct D2D communications in the Rayleigh channel [2, 3, 7, 8] and in the Suzuki channel [10] are not valid for the generalized fading such as Nakagami channel. Despite the formulations derived in [9] for the generalized fading can be used for the performance evaluation of direct D2D communications in Nakagami fading channel, it has complex and time-consuming expressions that does not make it applicable for solving the outage-based resource allocation optimization problems of direct D2D communications. In addition, the exact formulas obtained in [12-15], include some nonanalytic integrals, which introduce high complexity for evaluating the outage probability of underlay relay-aided D2D communications. On the contrary, the obtained low-complex closed-form expressions are just valid for high-enough SINRs. In addition, it is clear that the formulations and results of [11] are not applicable for relay-aided D2D communications in Nakagami fading channels and does not exist any low-complex outage probability expression for the DF relay-aided D2D communications appropriate for all SNRs in a Nakagami fading channel.

Energy efficiency as a crucial challenge in the future communication systems has become the main design target for $5 \mathrm{G}$ radio access networks. The high operational costs and/or impossibility of replacing or recharging wireless device batteries call for a new technology by which wireless devices can harvest energy from the environment via capturing ambient radio frequency (RF) signals, referred as energy harvesting (EH) $[16,17,18]$. The concept of simultaneous wireless information and power transfer (SWIPT) is gaining momentum among other EH approaches $[19,20,21]$ and modeling and performance evaluation of SWIPT-based direct/relay-aided D2D communications, as well as resource allocation and power control of these networks in fading channels, have recently attracted a lot of attention from the researchers [22-30].

The paper [22] investigated the resource allocation problem for D2D communications underlaying wireless powered communication networks by maximizing the sum throughput via joint time scheduling and power control. Ref. [23] focused on joint power control and spectrum resource allocation problem in SWIPT-based energy-harvesting D2D underlay networks. They formulated joint optimization problem as a 2D matching between D2D pairs and the cellular user equipment and proposed a preference establishment algorithm based on the Dinkelbach method and Lagrange dual decomposition. By considering the downlink resource reuse and energy harvesting, Ref. [24] maximized the sum-rate of the D2D links without degrading the quality of service (QoS) requirement of the cellular users. The authors of [24] formulated a sum-rate maximization problem of the joint resource block and power allocation for the D2D links, which resulted in a non-convex problem.

The resource allocation problem in terms of spectrum and energy under EH-powered D2D communications underlaying cellular network was investigated in [17]. A sum-rate maximization problem with consideration of QoS and available energy constraints was formulated, which is a non-polynomial (NP)-hard non-concave mixed-integer nonlinear programming (MINLP) problem. In [25], the authors investigated the tradeoff between the amount of energy used for relaying and the energy used for decoding the cellular user data at the relaying energy-harvested node. They formulated an NP-hard optimization problem to maximize the cellular user rate subject to a minimum D2D rate requirement constraint.

Ref. [26] proposed a SWIPT-based cooperative D2D network, where the $\mathrm{D} 2 \mathrm{D}$ pairs are distributed in a circular area. Expressions for the ergodic rates of single-hop and dual-hop scenarios in a Rayleigh fading channel were derived. Ref. [27] considered a D2D network with time-splitting protocol, where a D2D transmitter first harvests energy from a multiple-antenna power beacon and ambient RF sources and then uses the harvested energy to transmit data to the $\mathrm{D} 2 \mathrm{D}$ receiver. The authors of [27] derived analytical expressions for the average harvested energy, power outage probability, and the outage probability of the information transfer link, considering the effect of co-channel interference from homogeneous Poisson distributed interferes and the short-range propagation model for the path loss. Authors in [28] studied the outage performance of an AF-relay aided underlay D2D communications. Each relay node harvests the energy from the received RF signal and utilizes this energy to transfer the signal from one D2D user to the other D2D user. A power splitting-based relaying protocol was used at the relay to harvest the energy and to process the information. In particular, an exact outage probability expression under Rayleigh fading channels was derived, while an asymptotic outage expression was deduced in the high signal-to-noise ratio (SNR) regime. In [29], the authors investigated the outage probability of a SWIPT-based DF- 
relaying system in the presence of Nakagami fading. The derived closed-form outage expression was based on an infinite summation on Gamma and Hypergeometric regularized functions [30].

The above-mentioned research works show that joint resource allocation and power control in an energy-harvested direct/relay-aided D2D communications underlaying cellular network is an NP-hard problem. Most of these research works consider a Rayleigh channel and add a complexity for finding the power-splitting factor in the SWIPT scenario. Just Ref. [29] derived an SNR-based high-complex outage probability expression for a system consisting of three nodes in a Nakagami fading channel, which is not applicable for underlay D2D communications. It means that a low complexity closed-form expression for the outage probability (or throughput) of an $\mathrm{EH}-$ based underlay D2D communications in a general fading channel and a simple procedure to power saving and power splitting are needed.

In this research, first by considering a one-cell structure, a low-complexity closed-form outage probability formula for the direct underlay D2D communications is derived which is based on a combination of Gauss-Legendre quadrature and generalized Gauss-Laquerre quadrature numerical solutions. This solution has been used for solving non-analytic integrals, especially in works $[10,11$, and 31$]$. Then, by proposing a new idea for the energy harvested-based DF-relay-aided D2D communications in Nakagami channel, a low-complexity outage probability expression is extracted which is valid for both EH-based and non-EH-based scenarios. In summary, the following items are investigated in this research:

- Deriving a closed-form formula for the outage probability of the direct D2D communications in Nakagami channel.

- Extending the closed-form formulation of direct scenario for the DF-relay-assisted D2D communications.

- Proposing an idea to find a unique formulation for energyharvested and non-energy harvested scenarios.

- Suggesting a simple and low-complex procedure for power splitting in the SWIPT scenario applicable for both active and idle nodes.

The remainder of this paper is as follows. The system model for D2D communications underlaying cellular system is presented in Section 2. Section 3 founds a closed-form formula for the outage probability of underlay direct D2D communications in Nakagami channel. After power splitter design, Section 4 derives a closed-form expression for the outage probability of DF relay-aided scenario, which is valid for both energy-harvested and non-energy-harvested relayaided D2D communications. In Section 5, by obtaining the proper number of points in Gauss-Laguerre and generalized Gauss-Legendre solutions, low-complexity formulas are derived. Moreover, a comparative study between numerical analyses and simulation Monte-Carlo results are made. Finally, Section 6 concludes the paper.

\section{SYSTEM MODEL FOR UNDERLAY D2D COMMUNICATIONS}

According to Fig. 1, an omnidirectional BTS covers a circular cell. $M$ active and $M$ idle cellular users and $M$ D2D pairs are uniformly distributed throughout the cell. Each D2D pair can reuse only one radio resource of one active cellular user in the direct D2D communications between nearby TX and RX nodes of a D2D pair or with the help of a relay using an idle cellular user. As depicted in this figure, each active or idle cellular user and D2D pairs are equipped with an RF energyharvesting unit.

Each node can harvest the energy either it is in an active mode in a direct D2D/relay-assisted D2D/cellular mode or not. For the later one, the gathered energy can be saved in a battery useful for the times that the node is active. When it is active in

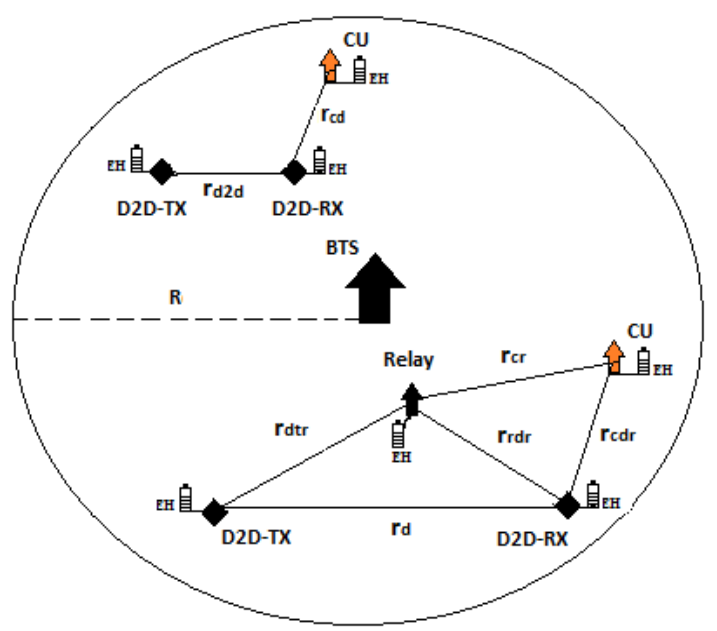

Fig. 1. The system model for energy-harvested direct/relay-aided D2D communications in a cell

a communications link, a part of the received energy goes to the energy harvesting section and the remaining part goes to the information processing and communications part. It means that when a node is not active, it harvests energy completely, while it uses most of the received signal including the desired RF signal, interference co-channel signal, and noise for the processing of information not for converting it to the electrical energy. In the not active case, the power-splitting unit completely connects the receiver to the energy harvesting part and saves the total received RF energy in the battery, which can be simply performed by a switch. In the active case, by fixing the transmit power of the relay node in the second hop, the complex optimization problem to find the optimum value for power splitting can be removed by a simple procedure as

- If the received signal in the relay node is greater than a switching threshold value, which depends on the required transmit power plus the power required for decoding and regenerating the signal by considering the efficiency of the energy harvesting system, it will be divided into two parts. According to this power-splitting scheme, someone can receive the acceptable signal in the decoding part, regenerate it, and manage both the signals to be received in the desired receiver and the co-channel interference induced in the cellular user in a joint manner.

- If the received signal in the relay node is lower than the above-mentioned switching threshold value, the total received signal goes to the information part and the saved energy in the battery helps the relay to process the signal and transmit it in the second hop with a pre-defined transmit 
power. In most of the times, this case occurs except the situation that the direct link between the nearby transmitter and receiver nodes of a D2D pair experiences a deep shadowing while the relay node has a strong line of sight links to both of them.

In order to have all parameters at a glance, symbols used in this paper and their descriptions are summarized in Table 1. [6]

Besides noise and path loss, Nakagami fading exists as (1)

$$
f_{R-N a k a g a m i}(r)=2\left(\frac{\mu}{\omega}\right) \frac{\mu}{\Gamma(\mu)} \exp \left(-\frac{\mu}{\omega} r^{2}\right) r>0
$$

Where $\Gamma(\mu)=\int_{0}^{\infty} t^{\mu-1} \exp (t) d t$ is a Gamma function for $0.5 \leq \mu<\infty$. The fading can be controlled by $\mu$ value, so the lower the $\mu$ equals to the deeper fading. For $\mu=0.5$, 1 , $1+k^{2} /(2 k+1)[32]$, Nakagami distribution respectively can be changed to one-side Gaussian, Rayleigh, and Rician distributions. $\mu \rightarrow \infty$, equals to a no fading channel with constant gain and no fluctuations [5]. Clearly, outage probability formula for Nakagami channel is a generalized form applicable for above-mentioned models.

\section{OUtAge Probability of Direct ScEnario}

In order to have a similar procedure for both energy harvested and non-energy harvested TX/RX nodes, it is assumed that there exist or harvested enough energy in the batteries to transmit and receive modes. SINR in the $i$ th D2D pair, which reuses the $j$ th cellular user's frequency channel, is

$$
\operatorname{SINR}_{i j}=\frac{P_{i i}\left|h_{d_{i}}\right|^{2}}{P_{i j}\left|h_{c_{j}}\right|^{2}+N}
$$

Where $P_{i i}=P_{d} \cdot r_{d 2 d}^{-\alpha}$ and $P_{i j}=P_{c} \cdot r_{c_{j} d_{i}}^{-\alpha}$ are receiving powers in a D2D-RX node, respectively due to D2D-TX node and cellular user. $P_{d}$ and $P_{c}$ are respectively the transmit powers of D2D-TX node and cellular user, $r_{d 2 d}$ is the distance between TX and RX nodes of a D2D pair, $r_{c_{j}} d_{i}$ is the distance between $i$-th D2D pair and $j$-th cellular user, $\left|h_{d_{i}}\right|^{2}$ and $\left|h_{c_{j}}\right|^{2}$ are the power gains of channels related to D2D pair and cellular users, $\alpha$ is the path loss, and $N$ is the noise power. Supposing $Z=X / Y, \quad$ so $\quad$ that $X=P_{i i}\left|h_{d_{i}}\right|^{2} \quad$ and $Y=P_{i j}\left|h_{c}\right|^{2}+N$ by considering [33 (equation 4-5)] and [33 (equation 5-8)], pdfs of non-negative random variables $X$ and $Y$ are as (3) and (4), respectively.
TABLE I

\begin{tabular}{|c|c|}
\hline Symbol & Description \\
\hline$P_{i i}$ & Received power in the i-th D2D-RX due to the i-th D2D-TX \\
\hline$P_{i j}$ & Received power in the i-th D2D-RX due to the j-th CU \\
\hline$P_{i i}^{\prime}$ & Received power in the $\mathrm{i}$-th relay due to the $\mathrm{i}$-th $\mathrm{D} 2 \mathrm{D}-\mathrm{TX}$ \\
\hline$P_{i j}^{\prime}$ & Received power in the $\mathrm{i}$-th relay due to the $\mathrm{j}$-th $\mathrm{CU}$ \\
\hline$P_{i i}^{\prime \prime}$ & Received power in the i-th D2D-RX due to the i-th relay \\
\hline$P_{i j}^{\prime \prime}$ & Received power in the i-th D2D-RX due to the $\mathrm{j}$-th $\mathrm{CU}$ \\
\hline$P_{d}$ & Transmit power of the D2D pair \\
\hline$P_{c}$ & Transmit power of the $\mathrm{CU}$ \\
\hline$P_{r}$ & Transmit power of the relay \\
\hline$P_{r E}$ & Electrical energy part of the received power of the relay \\
\hline$P_{r I}$ & Information part of the received power of the relay \\
\hline$\left|h_{d_{i}}\right|^{2}$ & Channel power gain of the i-th D2D pair \\
\hline$\left|h_{r_{i}}\right|^{2}$ & Channel power gain of the i-th relay \\
\hline$\left|h_{c j}\right|^{2}$ & Channel power gain of the $\mathrm{j}$-th $\mathrm{CU}$ \\
\hline$r_{d}$ & The distance between TX and RX nodes of a D2D pair \\
\hline$r_{d t_{i}} r_{i}$ & The distance between the D2D-TX node and relay \\
\hline$r_{r_{i} d r_{i}}$ & The distance between the relay and D2D-RX node \\
\hline$r_{c_{j} d r_{i}}$ & The distance between the CU and D2D-RX node \\
\hline$r_{c_{j}} r_{i}$ & The distance between the CU and relay \\
\hline$\sigma^{2}$ & The variance of white noise \\
\hline$N_{0} / 2$ & White noise power spectral density \\
\hline$N$ & Noise power \\
\hline$B W$ & Radio link bandwidth \\
\hline$\gamma_{t h}$ & Receiver threshold level \\
\hline$\alpha$ & Path loss exponent \\
\hline$M$ & Number of the active CUs, idle CUs and D2D pairs \\
\hline$m$ & Number of Gauss-Legendre points \\
\hline$n$ & Number of generalized Gauss-Laguerre points \\
\hline$\mu$ & Nakagami fading shape factor \\
\hline$\omega$ & Nakagami fading scale factor \\
\hline$\rho$ & Power splitting factor \\
\hline$\eta$ & Energy conversion efficiency \\
\hline$K$ & Number of Monte-Carlo simulation runs \\
\hline
\end{tabular}

A SUMMARY OF SYMBOLS AND THEIR DESCRIPTIONS 


$$
\begin{gathered}
f_{X}(x)=\frac{1}{2 \sqrt{P_{i i} \cdot x}} f_{R-N a k a g a m i}\left(\sqrt{\frac{x}{P_{i i}}}\right) \\
=\frac{\left(\frac{\mu}{\omega}\right) \mu}{\Gamma(\mu)} \frac{x^{\mu-1}}{P_{i i}{ }^{\mu}} \exp \left(-\frac{\mu}{\omega} \cdot \frac{x}{P_{i i}}\right) \\
f_{Y}(y)=\frac{1}{2 \sqrt{P_{i j}(y-N)}} f_{R-\text { Nakagami }}\left(\sqrt{\frac{y-N}{P_{i j}}}\right) \\
=\frac{\left(\frac{\mu}{\omega}\right)^{\mu}}{\Gamma(\mu)} \frac{(y-N)^{\mu-1}}{P_{i j}{ }^{\mu}} \exp \left(-\frac{\mu}{\omega} \frac{y-N}{P_{i j}}\right)
\end{gathered}
$$

Regarding [33 (equation 5-59)] and (3), (4), pdf of $Z$ is as

$$
f_{Z}(z)=\int_{0}^{\infty} k \cdot f_{X}(k z) f_{Y}(k) d k
$$

pdf of $Z$ can be attained by converting variable

$$
\begin{gathered}
t=k \frac{\mu}{\omega}\left(\frac{P_{i j} z+P_{i i}}{P_{i i} P_{i j}}\right), \text { as } \\
f_{Z}(z)=\left(\frac{2\left(\frac{\mu}{\omega}\right)^{\mu}}{\Gamma(\mu)}\right)^{2}\left(\frac{z}{P_{i i} P_{i j}}\right)^{\mu-1} \exp \left(-\frac{\mu N}{\omega P_{i j}}\right) \\
\times \int_{t=0}^{\infty}\left\{\frac{t^{\mu}}{\left(\frac{\mu}{\omega}\left(\frac{P_{i j} z+P_{i i}}{P_{i i} P_{i j}}\right)\right)^{\mu+1}}\left(\frac{\frac{\mu}{\omega}\left(\frac{P_{i j} z+P_{i i}}{P_{i i} P_{i j}}\right)}{\mathrm{N}}\right)\right. \\
\times \exp (-t)\} d t
\end{gathered}
$$

This non-analytic integral is in the form of $\int_{0}^{\infty} t^{\mu} f(t) \exp (-t) d t$. It can be solved by the generalized GaussLaguerre method using

$$
\begin{gathered}
\int_{0}^{\infty} t^{\mu} f(t) \exp (-t) d t=\sum_{V=1}^{n} S_{V} f\left(t_{V}\right) \\
L_{n}^{\mu}(x)=\sum_{V=0}^{n} \frac{(-1)^{v}}{v !}\left(\begin{array}{l}
n+\mu \\
n-v
\end{array}\right) x^{v} \\
S_{V}=\frac{\Gamma(\mu+n+1) x_{V}}{n !(n+1)^{2}\left(L_{n+1}^{\mu}\left(x_{V}\right)\right)^{2}}
\end{gathered}
$$

In (7), $x_{v}$ is the root of the generalized Gauss-Laguerre quadrature equation $\left(L_{n}^{v}\left(x_{v}\right)=0\right)$, which is given in (8), and $n$ is the number of points. The weights can be attained based on (9) [34]. Finally, (6) is given as (10) and general formulation for outage probability is as (11).

$$
\begin{gathered}
f_{Z}(z)=\left(\frac{2\left(\frac{\mu}{\omega}\right)^{\mu}}{\Gamma(\mu)}\right)^{2}\left(\frac{z}{P_{i i} P_{i j}}\right)^{\mu-1} \exp \left(-\frac{\mu N}{\omega P_{i j}}\right) \\
\times \sum_{v=1}^{n} s_{v} \frac{t_{v}^{\mu}}{\left(\frac{\mu}{\omega}\left(\frac{P_{i j} z+P_{i i}}{P_{i i} P_{i j}}\right)\right)^{\mu+1}}\left(\frac{t_{v}}{\frac{\mu}{\omega}\left(\frac{P_{i j} z+P_{i i}}{P_{i i} P_{i j}}\right)}-\mathrm{N}\right)^{\mu-1} \\
F_{Z}\left(\gamma_{t h}\right)=\int_{0}^{\gamma_{t h}} f_{Z}(z) d z
\end{gathered}
$$

Supposing $q=2 z / \gamma_{t h}-1$, (11) can be rewritten as (12). The integral in (12), $\int_{-1}^{+1} g(q) d q$ can be solved by the GaussLegendre method as (13)

$$
\begin{aligned}
& F_{Z}\left(\mathrm{y}_{\mathrm{th}}\right)=\left(\frac{2\left(\frac{\mu}{\omega}\right)^{\mu}}{\Gamma(\mu)}\right)^{2} \exp \left(-\frac{\mu N}{\omega P_{i j}}\right) \sum_{v=1}^{n} s_{v} \\
& \times \int_{-1}^{1}\left\{\frac{t_{v}^{\mu}}{\left(\frac{\mu}{\omega}\left(\frac{P_{i j} y_{t h}(q+1)+2 P_{i i}}{2 P_{i i} P_{i j}}\right)\right)^{\mu+1}}\right. \\
&\left.\times\left(\frac{y_{t h}(q+1)}{2 P_{i i} P_{i j}}\right)^{\mu-1}\left(\frac{t_{v}}{\frac{\mu}{\omega}\left(\frac{P_{i j} y_{t h}(q+1)+2 P_{i i}}{2 P_{i i} P_{i j}}\right)}-\mathrm{N}\right)^{\mu-1}\right\} d q \\
& \int_{-1}^{+1} g(q) d q=\sum_{r=1}^{m} S_{r} g\left(q_{r}\right)
\end{aligned}
$$

Where, $q_{r}$ is the root of the Legendre quadrature equation $\left(P_{m}(q)=0\right)$, given in (14), and $m$ is the number of points. The weights can be calculated as follows [35]:

$$
\begin{gathered}
P_{m}(x)=\frac{1}{2^{m} m !} \sum_{r=0}^{\left.\frac{m}{2}\right]}(-1)^{r} \times \frac{(2 m-2 r) !}{(m-r) !(m-2 r) !} x^{m-2 r} \\
S_{r}=\frac{2\left(1-q_{r}^{2}\right)}{(m+1)^{2}\left(P_{m+1}\left(q_{r}\right)\right)^{2}}
\end{gathered}
$$

Finally, the outage probability is given as

$$
\begin{aligned}
P_{\text {outage }}(n, m) & =\left(\frac{2\left(\frac{\mu}{\omega}\right)^{\mu}}{\Gamma(\mu)}\right)^{2}\left(\frac{y_{t h}\left(q_{r}+1\right)}{2 P_{i i} P_{i j}}\right)^{\mu-1} \exp \left(-\frac{\mu N}{\omega P_{i j}}\right) \\
& \times \sum_{v=1}^{n} \sum_{r=1}^{m} s_{v} s_{r} \times \frac{t_{v}^{\mu}}{\left(\frac{\mu}{\omega}\left(\frac{P_{i j} y_{t h}\left(q_{r}+1\right)+2 P_{i i}}{2 P_{i i} P_{i j}}\right)\right)^{\mu+1}} \\
& \times\left(\frac{t_{v}}{\frac{\mu}{\omega}\left(\frac{P_{i j} y_{t h}\left(q_{r}+1\right)+2 P_{i i}}{2 P_{i i} P_{i j}}\right)}-\mathrm{N}\right)^{\mu-1}
\end{aligned}
$$




\section{OUtAGE PROBABILITY FOR SWIPT-BASED DF RELAY- AIDED SCENARIO}

One of the main objectives in energy-harvested relay-aided D2D communications is optimum resource allocation, power control of D2D transmit nodes and selection of power splitting (PS) factor of relay nodes in a joint manner by minimizing the outage probability. Achieving a low-complexity closed-form expression for the outage probability helps us to do the optimization problem in an acceptable processing time. According to the results of the previous section, the outage probability of the energy-harvested SWIPT-based DF-relaying D2D communications underlaying cellular network in Nakagami fading channel can be found.

Consider the power splitting module at the relay, which splits the received signal in the relay node (17) by a PS factor, $\rho$, to two parts as (18) and (19), respectively for the energy harvesting and information modules [29],

$$
\begin{gathered}
P_{r r}=P_{i i}^{\prime} \cdot\left|h_{r_{i}}\right|^{2}+P_{i j}^{\prime} \cdot\left|h_{c j}\right|^{2}+N \\
P_{r_{E}}=\rho \cdot P_{r r} \\
P_{r_{I}}=(1-\rho) P_{r r}
\end{gathered}
$$

It is assumed that the transmit power of the relay node is fixed to a pre-defined value as (20) at the second time slot.

$$
\eta \cdot P_{r_{E}}=\eta \cdot \rho \cdot P_{r r}=\eta \cdot \rho\left(P_{i i}^{\prime} \cdot\left|h_{r_{i}}\right|^{2}+P_{i j}^{\prime} \cdot\left|h_{c}\right|^{2}+N\right)=P_{r}
$$

$\eta$ is the energy conversion efficiency of PS. Finally, yields

$$
\rho=\frac{P_{r}}{\eta\left(P_{i i}^{\prime} \cdot\left|h_{r_{i}}\right|^{2}+P_{i j}^{\prime} \cdot\left|h_{c_{j}}\right|^{2}+N\right)}
$$

In order to achieve the following features, the transmit power of the relay is fixed:

- Making it possible for the signals in the second hop to have the same power at all times in different states, whether the received signal in the first hop is strong or weak. When the signal received at the first hop is weak, the relay uses more energy stored in the battery, while the relay uses less battery energy for a strong received signal. Where the received signal in the relay is weak and no energy or low energy can be obtained, the quality of the signal in the second hop is such that the received signal in the first hop is strong. This ensures service uniformity and causes the first and second links to have a similar SINR. In this way, the final throughput will be increased, because it is determined by the weaker hop in the two-hop DF relay channels.

- Removing the complex optimization problem for jointly finding the optimum value of power splitting factor and controlling the transmit power of the relay using a simple procedure.

- Simplifying the power control process and choosing the splitting power factor in this way does not add any additional complexity to the computational complexity of the mode selection and resource allocation phases, which are timeconsuming enough.
- In the previous research works [36, 37], assuming the similar channel gains for the transmitter-relay and the relay-receiver links, it was concluded that the best relay is located between them in the middle. On this basis, the maximum throughput can be achieved when the relay power is at the same level as the transmit power of the D2D transmitter is. It is assumed that the selected relay provides both a lower delay and a higher throughput.

- In many studies, mode selection and resource allocation are performed while the transmit power of the transmitters are the same, and no power control is performed. In this way, without increasing the computational complexity for finding the proper power and splitting factor, the proposed method for previous non-energy harvested studies is valid, and the results of this research can be extended to the energy-harvested scenarios.

For the first time slot, $\rho$ value has no effect on the SINR and consequently the outage probability, because both the desired (signal from D2D transmit node) and non-desired (interference plus noise) parts are multiplied by $(1-\rho)$ as

$$
P_{r I}=(1-\rho) P_{r r}=(1-\rho) \cdot P_{i i}^{\prime} \cdot\left|h_{r_{i}}\right|^{2}+(1-\rho) \cdot\left(P_{i j}^{\prime} \cdot\left|h_{c j}\right|^{2}+N\right)
$$

In addition, the $\rho$ and $\eta$ values have no effect on the second time slot, because in accordance with the proposed idea mentioned above the transmit power of the relay node is fixed by using adaptive $\rho$ value in the first time slot.

In the DF relay-based D2D communications, the total SINR value depends on the minimum SINR of the first and second links, because the lowest SINR introduces the transmission rate. Considering a two-phase (two-hop) transmission scenario that the first phase is established between the D2D transmitter and the relay node and the second one is between the relay node and the D2D receiver, the achievable throughput can be easily modeled by the SINR value of the worst-case link, referred as the minimum SINR. If the first link supports the higher quality, it introduces higher throughput that cannot be transmitted correctly in the second link. Hence, the total achievable throughput in the final receiver (D2D RX) is equal to the achievable throughput in the second link. On the contrary, when the second link has a higher SINR than the first link, just the relay node receives, decodes and regenerates the data in the level of the first link's throughput, which is the maximum achievable throughput in the final receiver. Hence, the effective SINR in DF relaying scenario is as (23) including the SINRs of the first and second time slots as (24) and (25).

$$
\begin{gathered}
Z_{\text {tot }}=\operatorname{SINR}_{\text {tot }}=f_{D F}\left(Z_{1}, Z_{2}\right)=\min \left(Z_{1}, Z_{2}\right) \\
Z_{1}=\operatorname{SINR}_{h o p 1}=\frac{P_{i i}^{\prime}\left|h_{r_{i}}\right|^{2}}{P_{i j}^{\prime}\left|h_{c_{j}}\right|^{2}+N} \\
Z_{2}=\operatorname{SINR}_{h o p 2}=\frac{P_{i i}^{\prime \prime}\left|h_{r_{i}}\right|^{2}}{P_{i j}^{\prime \prime}\left|h_{c_{j}}\right|^{2}+N}
\end{gathered}
$$


Where, $P_{i i}^{\prime}=P_{d} \cdot r_{d t_{i} r_{i}}^{-\alpha}$ and $P_{i j}^{\prime}=P_{c} \cdot r_{c_{j} r_{i}}^{-\alpha}$ are receiving powers in the relay node, respectively due to the TX node of the D2D pair and the cellular user. $P_{i i}^{\prime \prime}=P_{r} \cdot r_{r_{i}}^{-\alpha} r_{i}$ and $P_{i j}^{\prime \prime}=P_{c} \cdot r_{c_{j}}^{-\alpha} d r_{i}$ are receiving powers in the $\mathrm{D} 2 \mathrm{D} \mathrm{RX}$ node, respectively due to the relay node and cellular user. $P_{d}, P_{c}$, and $P_{r}$ are transmit powers of the D2D TX node, cellular user, and relay node, respectively. $r_{d t_{i} r_{i}}, r_{r_{i} d r_{i}}, r_{c_{j} r_{i}}$, and $r_{c_{j} d r_{i}}$ are respectively the distance between $i$ th D2D TX node to $i$ th relay, $i$ th relay to $i$ th D2D RX node, $j$ th cellular user to $i$ th relay, and $j$ th cellular to $i$ th-D2D RX node. $\left|h_{r_{i}}\right|^{2}$ and $\left|h_{c_{j}}\right|^{2}$ are respectively the power gains of D2D-relay and cellular-relay (or cellular-D2D) channels, $\alpha$ is the path loss, and $N$ is the noise power.

The outage probability can be derived as follows:

$$
\begin{aligned}
P_{\text {out }}= & \mathrm{P}\left(Z_{t o t}<\gamma_{t h}\right)=P\left(\min \left(Z_{1}, Z_{2}\right)<\gamma_{t h}\right) \\
= & \mathrm{P}\left(Z_{1}<\gamma_{t h}, Z_{2} \geq \gamma_{t h}\right)+\mathrm{P}\left(Z_{2}<\gamma_{t h}, Z_{1} \geq \gamma_{t h}\right) \\
& +\mathrm{P}\left(Z_{1}<\gamma_{t h}, Z_{2}<\gamma_{t h}\right) \\
= & \mathrm{P}\left(Z_{1}<\gamma_{t h}\right) P\left(Z_{2}<\gamma_{t h}\right)+\mathrm{P}\left(Z_{1}<\gamma_{t h}\right)\left(1-\mathrm{P}\left(Z_{2}<\gamma_{t h}\right)\right) \\
& +\mathrm{P}\left(Z_{2}<\gamma_{t h}\right)\left(1-\mathrm{P}\left(Z_{1}<\gamma_{t h}\right)\right) \\
= & \mathrm{P}\left(Z_{1}<\gamma_{t h}\right)-\mathrm{P}\left(Z_{1}<\gamma_{t h}\right) \mathrm{P}\left(Z_{2}<\gamma_{t h}\right)+\mathrm{P}\left(Z_{2}<\gamma_{t h}\right) \\
& -\mathrm{P}\left(Z_{1}<\gamma_{t h}\right) \mathrm{P}\left(Z_{2}<\gamma_{t h}\right)+\mathrm{P}\left(Z_{1}<\gamma_{t h}\right) P\left(Z_{2}<\gamma_{t h}\right) \\
= & \mathrm{P}\left(Z_{1}<\gamma_{t h}\right)+\mathrm{P}\left(Z_{2}<\gamma_{t h}\right)-\mathrm{P}\left(Z_{1}<\gamma_{t h}\right) \mathrm{P}\left(Z_{2}<\gamma_{t h}\right) \\
= & F_{Z_{1}}\left(\gamma_{t h}\right)+F_{Z_{2}}\left(\gamma_{t h}\right)-F_{Z_{1}}\left(\gamma_{t h}\right) . F_{Z_{2}}\left(\gamma_{t h}\right)
\end{aligned}
$$

Where $F_{Z_{1}}\left(z_{1}\right)$ and $F_{Z_{2}}\left(z_{2}\right)$ are the cumulative distribution functions of the first and second time slots. For each time slot, there exists a direct mode. It means that equation (16) can be used for the outage probabilities of these paths.

\section{NUMERICAL ANALYSIS}

Herein, the proposed formulas (16) and (26) are analysed numerically and the results are compared to associated simulation results considering the parameters summarized in Table 2. All MATLAB simulations are run on a PC with $4 \mathrm{~GB}$ RAM, Processor: Intel (R) Core TM iS-2400 CPU at $3.10 \mathrm{GHz}$ on a 64-bit system.

In order to find a low-complexity formula or equivalently the appropriate number of points in Gauss-Laguerre and GaussLegendre expansions, respectively $n$ and $m$ in (7) and (14), the normalized mean square error (NMSE) defined as (27) is used.

$$
N M S E=\frac{1}{K} \sum_{k=1}^{K}\left(\frac{P_{\text {out }_{G L-G L}}(m, n)-P_{\text {out }_{\text {sim }}}(k)}{P_{\text {out }_{\text {sim }}}(k)}\right)^{2}
$$

Where, $P_{\text {out }}{ }_{G L-G L}(m, n)$ is the outage probability obtained from $(16), P_{\text {out }}(k)$ is the outage probability by $k^{\text {th }}$ run of simulation, and $K$ is the total number of simulation runs. It is clear that the lower number of coefficients in both GaussLaguerre and Gauss-Legendre solutions introduces the lower number of multiplications and additions, which means the lower computational complexity for the derived outage probability formulas.

In Fig. 2, NMSE between simulation results and numerical analyses for $\mu=0.5$ is plotted, where $n$ and $m$ are lower than 7 . It is clear that NMSE for $(n=1, m=4-7)$ is lower than that for the other cases with an NMSE lower than $5 \%$. It means that $n$ is more effective than $m$ for reducing the formula computational complexity.

In Fig. 3, NMSE is plotted in terms of $\mu$ and $m$, when $n=1$, which demonstrates that $(n, m)=(1,4)$ for $0.5 \leq \mu<0.7$ and $(n$, $m)=(1,2)$ for $\mu \geq 0.7$ are appropriate choices with an NMSE lower than $5 \%$.

TABLE II

SIMULATION PARAMETERS

\begin{tabular}{ll}
\hline \hline Parameter & Value \\
\hline Path loss exponent $(\alpha)$ & 4 \\
Cell radius $(R)$ & $1000 \mathrm{~m}$ \\
Number of D2D pairs $(M)$ & 10 \\
Number of active cellular users $(M)$ & 10 \\
Number of relays $(M)$ & $-159 \mathrm{dBm} / \mathrm{Hz}$ \\
Noise power spectral density $\left(N_{0} / 2\right)$ & $-30 \mathrm{~dB}$ \\
Pre-determined protection ratio $\left(\gamma_{t h}\right)$ & $20 \mathrm{dBm}$ \\
Transmit power of cellular user $\left(P_{c}\right)$ & $20 \mathrm{dBm}$ \\
Transmit power of relay node $\left(P_{r}\right)$ & $10 \mathrm{dBm}$ \\
Transmit power of D2D TX node $\left(P_{d}\right)$ & $50 \mathrm{~mm}$ \\
Signal bandwidth $(B W)$ & \\
\hline \hline
\end{tabular}

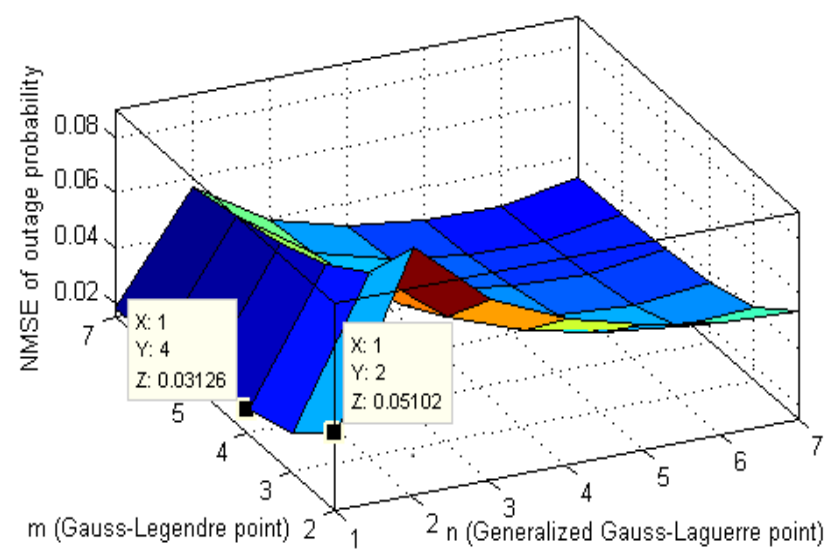

Fig. 2. NMSE of the outage probability for different (m, n). 


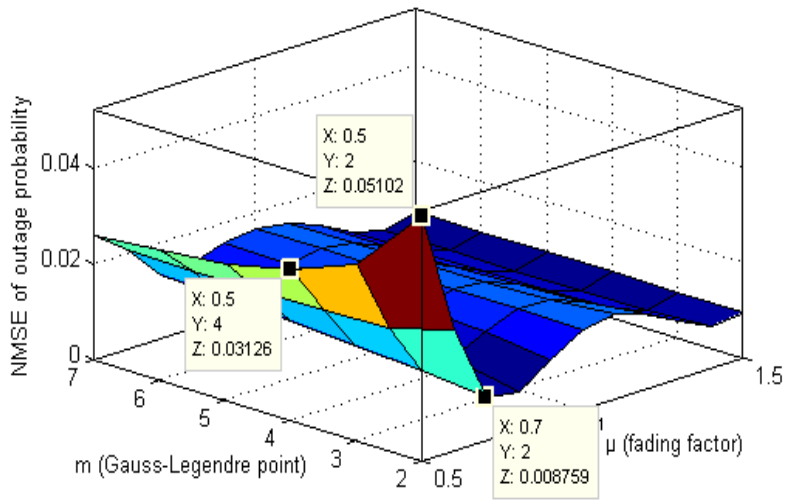

Fig. 3. NMSE of the outage probability for different $(\mathrm{m}, \mu)$

In Fig. 4, considering the results of Fig. 3, the outage probabilities of the direct D2D communications for appropriate values of $m$ and $n$ in $\mu=0.5$ and $\mu=1$ are plotted. As depicted in Fig. 4, the results are valid for all distances between D2D pair and cellular user. Furthermore, increasing the average distance between the D2D pair and the cellular user is the reason for decreasing the outage probability. In other words, increasing the average distance between the D2D pair and the cellular user introduces lower interference between them which means the probability that signal to interference plus noise ratio is above the threshold level is increased or equivalently the outage probability is decreased.

Thus, according to Figs 2-4, in the direct mode, equation (16) can be simplified as (28). In the DF-relay aided scenario, applying the equation (28) for both time slots 1 and 2, concludes the closed-form expression for the outage probability as equation (26), where $F_{Z_{1}}\left(z_{1}\right)$ and $F_{Z_{2}}\left(z_{2}\right)$ are as

$$
\begin{aligned}
& F_{Z}\left(\gamma_{t h}\right)=2 P_{i i} P_{i j}\left(\frac{1}{\Gamma(\mu)}\right)^{2} \gamma_{t h}^{\mu} \exp \left(\frac{\mu}{\omega} \cdot \frac{N}{P_{i j}}\right) \times \sum_{r=1}^{m} S_{r} \\
& \times\left\{\frac{\left(\left(q_{r}+1\right)\left(2 P_{i i} P_{i j}-\frac{\mu}{\omega} N\left(P_{i j} \gamma_{t h}\left(q_{r}+1\right)+2 P_{i i}\right)\right)\right)^{\mu-1}}{\left(P_{i j} \gamma_{t h}\left(q_{r}+1\right)+2 P_{i i}\right)^{2 \mu}}\right\} \\
& F_{Z_{2}}\left(\gamma_{t h}\right)=2 P_{i i}^{\prime} P_{i j}^{\prime}\left(\frac{1}{\Gamma(\mu)}\right)^{2} \gamma_{t h}^{\mu} \exp \left(\frac{\mu}{\omega} \cdot \frac{N}{P_{i j}^{\prime}}\right) \times \sum_{r=1}^{m} S_{r} \\
& \times\left\{\frac{\left(\left(q_{r}+1\right)\left(2 P_{i i}^{\prime} P_{i j}^{\prime}-\frac{\mu}{\omega} N\left(P_{i j}^{\prime} \gamma_{t h}\left(q_{r}+1\right)+2 P_{i i}^{\prime}\right)\right)\right)^{\mu-1}}{\left(P_{i j}^{\prime} \gamma_{t h}\left(q_{r}+1\right)+2 P_{i i}^{\prime}\right)^{2 \mu}}\right\}
\end{aligned}
$$

In Fig. 5, the outage probabilities of direct and DF-relay aided scenarios are plotted in two cases, $(n, m, \mu)=(1,4,0.5)$ and $(n, m, \mu)=(1,2,1)$. As shown, there exists a close agreement between the results of numerical analyses (eqs. 16, 26) and associated simulations for different average distances between transmit and receive nodes of a D2D pair. It is clear that direct D2D communications is the best mode if the TX-RX distances are smaller than $120 \mathrm{~m}$ and $140 \mathrm{~m}$, respectively in the cases of $\mu=1, \mu=0.5$. For distances greater than $120 \mathrm{~m}$ and $140 \mathrm{~m}$, DF relay-aided scenario offers lower outage probabilities with respect to the direct one. Finally, it is clear that increasing the Nakagami fading shape factor is the reason for decreasing the outage probability. Figures 4 and 5 show that in a dynamic range of $80 \mathrm{~dB}$ for the outage probability, the numerical values extracted from the derived Gauss-Laguerre/Gauss-Legendre formulas are close to the simulation results. By increasing the fading shape factor, the fluctuations of the signal strength reduce which means the lower fading depth compared to that for the lower values of $\mu$. Increasing the fading shape factor makes narrower probability density function concentrating around the peak value and shifting the pdf peak value to the larger power values which both of them directly decrease the depth of fading and the probability that the received signal goes under a threshold value, referred as outage probability.

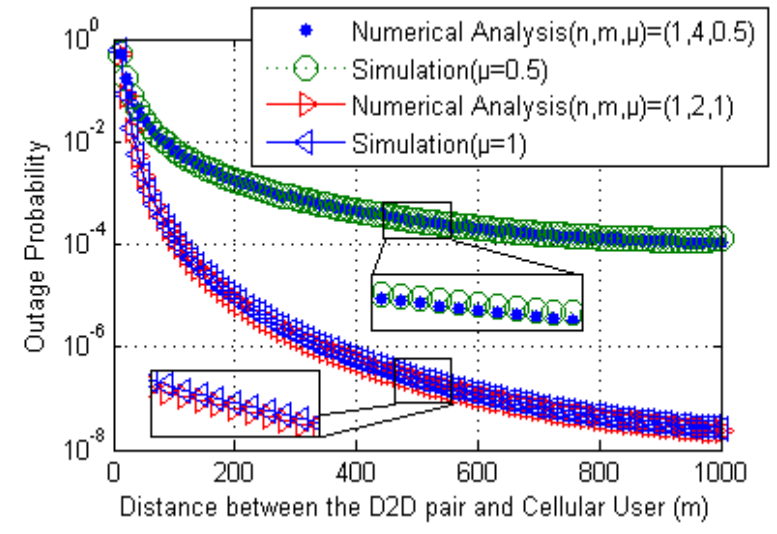

Fig. 4. The outage probability vs. the D2D-CU distance

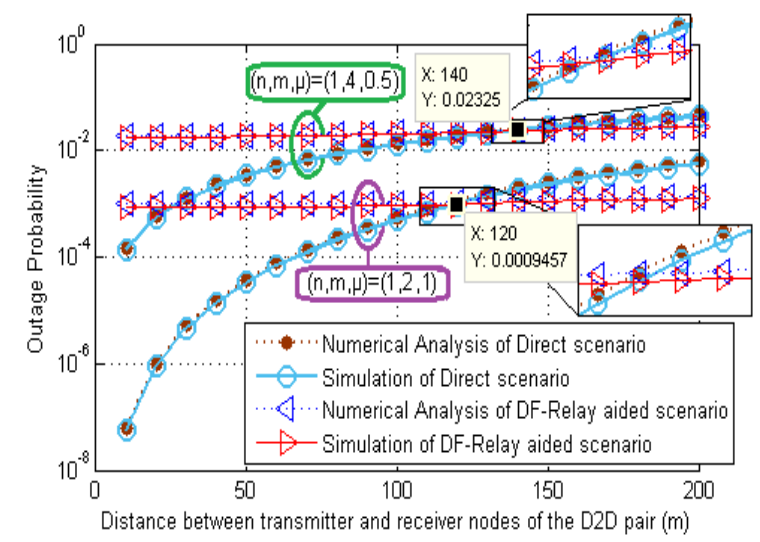

Fig. 5. The outage probability vs. the average distance between the transmitreceive nodes of a $\mathrm{D} 2 \mathrm{D}$ pair

This investigation is focused on the underlay D2D communications when the traffic load of the cellular users is high and the base station is not able to support the requests by cellular mode. Hence, the cellular users use all the assigned frequency channels and the D2D links in an interferencecontrolled scenario can be made to handle the requests. In other words, the maximum number of D2D users in the one-reuse scenario (as mentioned in the system model) equals the number of active cellular users. If the number of D2D users is lower than this value, some frequency channels are not reused with any impact on the outage probability of D2D users, which are active in the other reused frequency channels because the frequency channels are orthogonal. Therefore, the extracted outage probability formula of this research work is valid for the 
case that the number of D2D requests is lower than or equal to the number of active cellular users. For the case that the number of $\mathrm{D} 2 \mathrm{D}$ requests is greater than the number of active cellular users, there are two solutions as follows:

- For the one-reuse scenario, investigated in this paper, up to $M$ D2D pairs will be served and the extra ones will be blocked. For this case, the outage probability just evaluates the performance of active links and the performance analysis for all D2D requests may be evaluated by blocking probability and/or successful probability.

- The extracted outage probability formulas for the one-reuse scenario are not valid for more than one reuse scenario (not considered in this research), because the effect of more than one interference for each D2D user should be considered.

\section{CONCLUSIONS}

Based on the Gauss-Legendre/Gauss-Laguerre solutions, a closed-form formula for the outage probability of underlay DF relay-aided D2D communications in Nakagami fading channel was obtained. By designing the power splitter module such that the transmit power of the relay node is fixed to a pre-defined value, it was shown that the closed-form expression for the outage probability of DF relay-aided D2D communications in the case of SWIPT-based energy-harvested relaying scenario is the same as non-harvested one. Numerical analyses indicated that in higher $n$ (number of generalized Gauss-Laguerre points) and $m$ (number of Gauss-Legendre points), the proposed models for the outage probability show an excellent agreement with the associated simulation results. It was concluded that ( $n$, $m)=(1,4)$ for $0.5 \leq \mu<0.7$ and $(n, m)=(1,2)$ for $\mu \geq 0.7$, offer the best approximations. It should be noted that for optimizing the resource (frequency channel and power) allocation and mode selection problem, it is needed to minimize the outage probability subject to some constraints. By the results of this research and using the proposed low-complexity formulations of the outage probability for the Nakagami fading channel, the optimization problem will be solved in a time-efficient manner.

In this investigation, we assumed that the required energy for a node can be completely delivered by a rechargeable battery or it can be harvested in an active communications based on a SWIPT method. The average harvested power and total saved energy in the battery depends on the physical (the number and density of active transmitters, background noise), propagation (the type of channel in the view of path loss and fading components), and traffic (the number of requests and the average time for a communications) conditions. These parameters affect the quality of service and network lifetime, which can be investigated as a future work.

\section{ACKNOWLEDGMENT}

I would like to thank Mr. Hossein Ghavami, Shahid Rajaee Teacher Training University, for the help he provided.

\section{REFERENCES}

[1] S. Shirvani Moghaddam, "A novel resource reuse in device to device communications," Paper presented at: Internet Technologies and Society (ITS) Conference, 2017: 35-42; Sydney, Australia.

[2] S. Wang, W. Guo, Z. Zhou, Y. Wu, and X. Chu, "Outage probability for multi-hop D2D communications with shortest path routing," IEEE
Commun. Lett., vol. 19, no. 11, pp. 1997-2000, 2015, DOI: 10.1109/LCOMM.2015.2475428.

[3] J. Liu, H. Nishiyama, N. Kato, and J. Guo, "On the outage probability of device-to-device-communication-enabled multichannel cellular networks: An RSS-threshold-based perspective," IEEE J. Select. Areas Commun., vol. 34, no. 1, pp. 163-175, 2016, DOI: 10.1109/JSAC.2015.2452492.

[4] V. Aalo, C. Mukasa, and G. Efthymoglou, "Effect of mobility on the outage and BER performances of digital transmissions over Nakagami-m fading channels," IEEE Trans. Veh. Technol., vol. 65, no. 4, pp. 27152721, 2016, DOI: 10.1109/TVT.2015.2421502.

[5] C. Tang, G. Pan, and T. Li, "Secrecy outage analysis of underlay cognitive radio unit over Nakagami-m fading channels," IEEE Wireless Commun. Lett., vol. 3, no. 6, pp. 609-612, 2014, DOI: 10.1109/LWC.2014.2350501.

[6] P.M. Shankar, "Fading and Shadowing in Wireless Systems," SpringerVerlag, 2012.

[7] H. Ghavami, S. Shirvani Moghaddam, "Analysis of outage probability for in-band device to device Communications underlaying cellular network," International J. Commun. Sys. (IJCS), vol. 30, no. 14, pp. 1-8, 2017, DOI: $10.1002 /$ dac. 3286

[8] K.M. Saidul Hug, S. Momtaz, and J. Rodriquez, "Outage probability analysis for device-to-device system," Paper presented at: The IEEE International Conference on Communications (ICC), 2016; Kuala Lumpur, Malaysia, DOI: 10.1109/ICC.2016.7510678.

[9] Y.J. Chun, S. Cotton, H. Dhillon, A. Ghrayeb, and M. Hasna, "A stochastic geometric analysis of device-todevice communications operating over generalized fading channels," IEEE Trans. Wireless Commun., vol. 16, no. 7, pp. 4151-4165, 2017, DOI: 10.1109/TWC.2017.2689759.

[10] H. Ghavami, S. Shirvani Moghaddam, "Outage probability of device to device communications underlaying cellular network in Suzuki fading Channel," IEEE Commun. Lett., vol. 21, no. 5, pp. 1203-1206, 2017, DOI: 10.1109/LCOMM.2017.2655042.

[11] S. Shirvani Moghaddam, "Outage analysis of non-orthogonal in-band relay-aided device-to-device communications in Rayleigh fading channels. Internet Technol. Lett. (ITL), vol. 1, no. 2, 2018, DOI: 10.1002/itl2.8

[12] C. Zhong, T. Ratnarajah, and K.K. Wong, "Outage analysis of decodeand-forward cognitive dual-hop systems with the interference constraint in Nakagami-m fading channels," IEEE Trans. Veh. Technol., vol. 60, no. 6, pp. 2875-2879, 2011, DOI: 10.1109/TVT.2011.2159256.

[13] X. Zhang, Y. Zhang, Z. Yan, J. Xing, and W. Wang, "Performance analysis of cognitive relay networks over Nakagami-m fading channels," IEEE J. Select Areas Commun., vol. 33, no. 5, pp. 865-877, 2015, DOI: 10.1109/JSAC.2014.2361081.

[14] Y. Ni, S. Jin, R. Tian, K.K. Wong, H. Zhu, and S. Shao, "Outage analysis for device-to-device communication assisted by two-way decode-andforward relaying," Paper presented at: International Conference on Wireless Communications and Signal Processing; 2013; Hangzhou, China, DOI: 10.1109/WCSP.2013.6677242.

[15] B. Zhong, J. Zhang, Q. Zeng, and X. Dai, "Coverage probability analysis for full duplex relay aided device-to-device communications networks," China Commun., vol. 13, no. 11, pp. 60-67, 2016, DOI: 10.1109/CC.2016.7781718.

[16] H. Wang, J. Wang, G. Ding, L. Wang, T.A. Tsiftsis, and P.K. Sharma, "Resource allocation for energy harvesting-powered D2D Communication underlaying UAV-assisted networks," IEEE Trans. Green Commun. Netw., vol. 2, no. 1, pp. 14-24, 2018, DOI: $10.1109 /$ TGCN.2017.2767203.

[17] Y. Luo, P. Hong, R. Su, and K. Xue, "Energy-efficient scheduling and power allocation for energy harvesting-based D2D communication," Paper presented at: IEEE Global Communication Conference (GLOBECOM), 2017:1-6; Singapore, DOI: 10.1109/GLOCOM.2017.82 54193.

[18] J. Huang, C.-C. Xing, and C. Wang, "Simultaneous Wireless Information and Power Transfer: Technologies, Applications, and Research Challenges," IEEE Communications Magazine, vol. 55, no. 11, pp. 26-32, 2017, DOI: 10.1109/MCOM.2017.1600806.

[19] R. Jiang, K. Xiong, P. Fan, and Z. Zhong, "Outage performance of SWIPT-enabled two-way relay networks," Paper presented at: International Workshop on High Mobility Wireless Communications (HMWC), 2015:106-110; Xi'an, China, DOI: 10.1109/HMWC.2015.735 4345. 
[20] F. Jameel, A. Ali, and R. Khan, "Optimal time switching and power splitting in SWIPT," Paper presented at: 19th International Multi-Topic Conference (INMIC), 2016:1-5; Islamabad, Pakistan, DOI: $10.1109 /$ INMIC.2016.7840157.

[21] H. Lee, C. Song, S.H. Choi, and I. Lee, "Outage probability analysis and power splitter designs for SWIPT relaying systems with direct link," IEEE Commun. Lett., vol. 21, no. 3, pp. 648-651, 2017, DOI: 10.1109/LCOMM.2016.2627055.

[22] H. Wang, J. Wang, G. Ding, and Z. Han, "D2D communications underlaying wireless powered communication networks," IEEE Trans. Veh. Technol., vol. 67, no. 8, pp. 7872-7876, 2018, DOI: 10.1109/TVT.2018.2832068.

[23] Z. Zhou, C. Gao, C. Xu, T. Chen, D. Zhang, and S. Mumtaz, "Energyefficient stable matching for resource allocation in energy harvestingbased device-to-device communications," IEEE Access, vol. 5, pp. 1518415196, 2017, DOI: 10.1109/ACCESS.2017.2678508.

[24] S. Gupta, R. Zhang, and L. Hanzo, "Energy harvesting aided device-todevice communication underlaying the cellular downlink," IEEE Access, vol. 5, pp. 7405-7413, 2017, DOI: 10.1109/ACCESS.2016.2600242.

[25] M. Seif, A. El-Keyi, K.G. Seddik, and M. Nafie, "Cooperative D2D communication in downlink cellular networks with energy harvesting capability," Paper presented at: $13^{\text {th }}$ International Communications and Mobile Computing Conference (IWCMC), 2017; Valencia, Spain, DOI: 10.1109/IWCMC.2017.7986283.

[26] R.I. Ansari, S.A. Hassan, and C. Chrysostomou "A SWIPT-based deviceto-device cooperative network," Paper presented at: $24^{\text {th }}$ International Conference on Telecommunication (ICT), 2017, Limassol, Cyprus, DOI: 10.1109/ICT.2017.7998285.

[27] T.X. Doan, T.M. Hoang, T.Q. Duong, and H.Q. Ngo, "Energy harvestingbased D2D communications in the presence of interference and ambien RF sources," IEEE Access, vol. 5, pp. 5224-5234, 2017, DOI 10.1109/ACCESS.2017.2681696.

[28] D. Agrawal, S. Yanav, "Outage performance of energy harvesting relayassisted device-to-device communication," Paper presented at: International Conference on Wireless Communications, Signal Processing and Networking (WiSPNET), 2017, Chennai, India, DOI: 10.1109/WiSPNET.2017.8299763.

[29] F. Nawaz, S.A. Hassan, S. Aissa, and S. Saleem, "Outage probability for a decode-and-forward SWIPT relaying system in Nakagami fading," Internet Technol. Lett., vol. 1, no. 1, e13, 2018, DOI: 10.1001/it12.13.

[30] M. Abramowitz, "Handbook of Mathematical Functions, with Formulas, Graphs, and Mathematical Tables," Milwaukee, WI: Dover Publications, 1974.

[31] N. Mastronarde, V. Pate, J. Xu, L. Liu, and M. van der Schaar, "To relay or not to relay: Learning device-to-device relaying strategies in cellular networks," IEEE Trans. Mobile Comput., vol. 15, no. 6, pp. 1569-1585, 2016, DOI: 10.1109/TMC.2015.2465379.

[32] M. Nakagami, "The m-distribution-A General Formula of Intensity Distribution of Rapid Fading, " In W. C. Hoffman: Statistical Methods of Radio Wave Propagation, Oxford, England, 1960.

[33] A. Papoulis and S.U. Pillai, "Probability, Random Variables, and Stochastic Processes," McGraw-Hill, 2002.

[34] H. Cohen, "Numerical Approximation Methods," Springer, 2011.

[35] C. Pozrikidis, "Numerical Computation in Science and Engineering," Oxford University Press, 2008

[36] S. Shirvani Moghaddam, F. Baramaki Yazdi, "Bit error rate evaluation for different relay selection schemes in decode-and-forward two-way relay networks," International Journal of Research Studies in Electrical and Electronics Engineering (IJRSEEE), vol. 1, no. 1, pp. 35-41, 2015.

[37] S. Shirvani Moghaddam, F. Baramaki Yazdi, "A new modification on relay selection by considering the effect of relay occupancy," Journal of Scientific Research and Reports (JSRR), vol. 4, no. 3, pp. 241-252, 2015, DOI: $10.973 / J S R R / 2015 / 13002$.

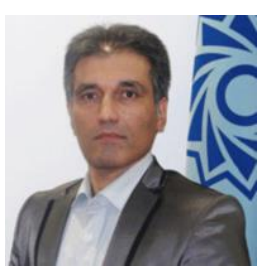

S. Shirvani Moghaddam was born in KhorramAbad, Iran, in 1969. He received the B.Sc. and M.Sc. degrees, both in Electrical Engineering, in 1992 and 1995, respectively. He also received the Ph.D. degree in Electrical Engineering from Iran University of Science and Technology (IUST), Tehran, Iran, in 2001. Since 2003, he has been with the Faculty of Electrical Engineering, Shahid Rajaee Teacher Training University (SRTTU), Tehran, Iran. Currently, he is an associate professor of SRTTU and a senior member of the IEEE. According to more than 140 international Journal and conference papers, 2 books on digital communications, one book chapter on MIMO systems, and the editor of one book entitled "Cognitive radio in 4G/5G wireless communication systems," his research interests include cognitive radio, D2D communications, power control and beamforming in cellular networks, handover in HetNets, compressive sensing, adaptive array processing, and DOA estimation. 